\title{
Anemia and Related Factors Among Highly Active Antiretroviral Therapy Experienced Children in Hawassa Comprehensive Specialized Hospital, Southern Ethiopia: Emphasis on Patient Management
}

This article was published in the following Dove Press journal:

Drug, Healthcare and Patient Safety

\section{Demissie Assegu Fenta (DID Metsihet Mohammed Nuru' Tilahun Yemane ${ }^{2}$ Yaregal Asres ${ }^{3}$ Temesgen Bizuayehu Wube (D)}

'School of Medical Laboratory, College of Medicine and Health Sciences, Hawassa University, Hawassa, Ethiopia; ${ }^{2}$ School of Medical Laboratory Sciences, Faculty of Health Sciences, Jimma University, Jimma, Ethiopia; ${ }^{3}$ Department of Medical Laboratory, College of Medicine and Health Science, Baherdar University, Baherdar, Ethiopia
Correspondence: Demissie Assegu Fenta School of Medical Laboratory, College of Medicine and Health Sciences, Hawassa University, Hawassa, Ethiopia

Tel +251911020189

Email demissieassegu@yahoo.com
Background: Human Immunodeficiency Virus (HIV) and its therapy cause a variety of hematological abnormalities that have been known to be one of the most common causes of morbidity and mortality in HIV-positive children. One of the commonly observed hematologic manifestations in HIV-positive children is anemia and it has a multifactorial source. We intended to assess the prevalence, as well as its related factors of anemia among Highly Active Antiretroviral Therapy (HAART), experienced children.

Methods: A hospital-based cross-sectional study was employed at Hawassa comprehensive specialized hospital from February 15-June 15, 2018. Overall, 273 HAART-practiced children were included in the study. Socio-demographic variables and clinical data were collected using a standard and pretested questionnaire. Medical records were reviewed for each study participant using a standard checklist. Blood specimens were collected and examined for complete blood count, CD4 cell count and blood film for hemoparasites and morphological classification of anemia, whereas stool specimens were collected and examined for intestinal parasites. Data were entered into Epidata and transferred to SPSS (Statistical Package for Social Science) version 20 software. Descriptive analysis was done for prevalence and binary and multivariate logistic regression was used to determine factors associated with anemia. Statistical significance was stated at $\mathrm{P}$-value $<0.05$.

Results: The overall prevalence of anemia in this study was $11.4 \%$. Morphologically the predominant anemia was Normocytic Normochromic anemia which accounted for $64.5 \%$. In the current study, children within the age group of $<7$ years (AOR: 3, CI: 1.2-7.5, $\mathrm{P}=0.02$ ), those who were rural residents (AOR: 2.6, CI: 1.0-6.6, $\mathrm{P}=0.042$ ) and those with viral load $>150 \mathrm{copies} / \mathrm{mL}$ (AOR: 3.4, CI: 1.36-8.3, $\mathrm{P}=0.009$ ) were found to be significantly associated with anemia.

Conclusion: The prevalence of anemia in this study was $11.4 \%$. It was significantly associated with different factors such as age, residence and viral load. Therefore, regular follow-up management should be emphasized for HAART-experienced children. Hence, there is a need for a longitudinal study to be conducted further to explore the causes of anemia due to HIV and the pattern of hemoglobin changes with HAART- experienced children will be very important.

Keywords: anemia, children, HIV, rural residents, viral load, HAART, Hawassa, Ethiopia

\section{Introduction}

Hematological abnormalities have been known to be the most common cause of morbidity and mortality in Human immunodeficiency virus (HIV)-positive people receiving 
HAART including children. ${ }^{1,2}$ HIV continues to affect mainly vulnerable populations such as children and pregnant women with a profound impact on disease progression and morbidity and mortality. ${ }^{1,2}$ Anemia remains the most commonly observed hematological abnormality and an independent predictive indicator of HIV disease progression in people living with HIV particularly in children.,

The root cause of anemia in HIV-positive people receiving HAART is multifactorial in its source. Infiltrations of the bone marrow by neoplasm or infection, practice of myelosuppressive therapy, reduction in the production of endogenous erythropoietin, hemolysis that may result from red blood cells (RBC) autoantibodies, influence of opportunistic infection hemophagocytic syndrome, hematinic deficiency, drug toxicity, chronic immune activation and inflammation, iron deficiency combined anti-retroviral therapy (cART) $)^{5,6}$ and direct or indirect consequence of HIV-related lymphomas are some of the causes of anemia in HIV-positive people. ${ }^{7,8}$ Currently, numerous attempts have been commenced to elucidate the mechanisms leading to HIV-related anemia. ${ }^{9}$ In-vitro data recommended that HIV itself may reduce erythropoiesis through apoptosis of Erythroid precursors or infection of auxiliary cells, by shifting cytokine and erythropoietin reactions. Unlike adults, children may be more vulnerable to these mechanisms for the reason that they differ from adults in their hematopoiesis, increased cytokine reactions, or viral load ${ }^{10}$.

Several studies revealed that anemia is related to reduced survival and increased disease progression in HIV infection. In children, it might present with weakness, fatigue, tachypnea, congestive cardiac failure, and poor mental status, social-emotional and neurophysiologic functioning. ${ }^{10}$ An affirmative relationship has also been reported between the prevalence of anemia and the severity of the clinical disease, its common complication of pediatric HIV infection, and its association with a poor prognosis. ${ }^{11}$

In addition to HIV infection, anemia prevalence in children can also be affected by the clinical stage of HIV, sex, age, race, height, weight, and concurrent illness as well as the definition of anemia used ${ }^{12}$ The prevalence of anemia in HIV-infected children in resource-limited countries might be attributed to the fact that many of the children in these regions are also iron deficient which is accompanied by poor socioeconomic status and nutrition. ${ }^{12}$

Children infected with HIV revealed a healthy growth in height and weight during the HAART experience, comprising of those who failed to completely suppress the virus. ${ }^{13}$ Older children initiating HAART with severe immune suppression were less likely to achieve a successful treatment outcome, emphasizing the importance of initiating HAART early to ensure adequate immune and growth responses. ${ }^{13}$ In spite of the direct effect of HIV, HAART-therapy also causes anemia and different adverse drug reactions. ${ }^{14,15}$ HAART can reduce the incidence of anemia and lymphopenia by suppressing viral replication and increasing CD4 cell count. However, anemia remains a common problem for patients treated with prophylactic and antiretroviral drugs. Drugs such as cotrimoxazole and zidovudine (AZT) often result in reticuloendothelial iron block which in turn reduces the production of erythrocytes. ${ }^{16}$

The risk of HIV-related anemia in resource-limited countries such as Ethiopia might be different from developed countries due to the persistence of factors like malnutrition, tuberculosis, and parasitic infections. However, in Ethiopia, there were few studies conducted on the association of anemia and HAART-therapy among HIV-positive children which is one of the predictors of virological treatment failure among children and needs emphasis during treatment. Therefore, this study was aimed at evaluating anemia and its associated factors among children on HAART at the Pediatric ART-clinic of Hawassa University Comprehensive Specialized Hospital, Hawassa Southern, Ethiopia.

\section{Materials and Methods Study Setting and Population}

A hospital-based, cross-sectional study was conducted at Hawassa University College of Medicine and Health Science Comprehensive Specialized Hospital from February to June 2018. All 273 registered HAART-experienced children were included at the hospital pediatric ART-clinic during the study period. The study participant's parent or guardian was informed about the purpose of the study and written informed consent and assent from each child was obtained before the administration of the questionnaire and sample collection included this study. However, children who were on treatment for anemia in the last 3 months, taking nutrient supplementation for the last 1 year, HAART-experienced children who took HAART for less than 3 months and those who had transfusion treatment within 3 months of data collection were excluded. Socio-demographic variables and clinical characteristics were collected using a standard pretested questionnaire at the interview. Medical records for each study participant were reviewed using the standard checklist. 


\section{Collection and Laboratory Analysis}

Data were collected by trained nurses using a standard and pre-tested questionnaire during the participants' respective appointment time for follow up. From each participant 3-5 mL of venous blood was collected using an EDTA vacutainer tube by an expert laboratory technologist and sent to the hematology laboratory. The blood samples were used for complete blood count (hemoglobin, Hematocrit, mean cell volume (MCV), mean cell hemoglobin $(\mathrm{MCH})$, mean cell hemoglobin concentration (MCHC) and Red Blood Cell count (RBC) using hematological autoanalyzer (Ruby Cell-Dyne 3000 USA) within 30 mins after collection. CD4+ T-lymphocyte cell count was also performed using the Becton Dickenson (BD) FACS count (BD, USA) and both thin and thick blood film was prepared for microscopic examination of hemoparasites and morphological studies of anemia by expert laboratory technologist. One gram of stool sample was also collected from each participant using a standard stool cup and sent to the medical parasitology laboratory and examined for intestinal parasitic infection using wet mount preparation.

World Health Organization (WHO) $\mathrm{Hb}$ reference range was used to classify the Hb level as anemic or normal based on the $\mathrm{Hb}$ value of the participants and assigned in one of the following categories: $<11.0 \mathrm{~g} / \mathrm{dl}$ for children $<5$ years old, $<11.5 \mathrm{~g} / \mathrm{dl}$ for children $5-11.9$ years old and $<12.0 \mathrm{~g} / \mathrm{dl}$ for children $12-14.9$ years old after adjustment of altitude and other factors. ${ }^{4}$

\section{Quality Control}

The quality of the data was assured by strict follow up of the standard operating procedures (SOP) for specimen collection, reagent checkup and usage of manufacturer's instructions. Control reagents were used to check the accuracy and precision of hematology parameters, CD4 T-lymphocyte cells, and viral load numbers. For the morphological study, a control smeared slide was used to check the quality of the Giemsa solution reagent.

\section{Statistical Analysis}

Data were entered into Epidata and transferred to SPSS version 20. Descriptive statistics were used to analyze the frequency of anemia and other descriptive variables. Bivariate and multivariate regression was used to identify independent predictors of anemia. Categorical variables were analyzed using chi-square tests or Fisher exact test.
In all cases, statistical significance was determined if the p-value was no greater than 0.05 .

\section{Ethical Clearance}

Ethical clearance was obtained from Jimma University Institute of Health Science and Hawassa University College of Medicine and Health Science Institutional review board. Consent was taken from their parents or guardians on behalf of children less than 7 years of age and ascent and written consent was received from both parents and guardians for children with the age range of 7-14 years before enrollment into the study. The confidentiality of the data was kept. Those results which required further management were reported to the clinician working in the ART-clinic of the hospital.

\section{Results \\ Socio-Demographic and Clinical Features of the Study Population}

Overall, 273 HAART-experienced children were enrolled in this study, with 139 (50.9\%) being females. The mean (standard deviation, SD) age was 10.2 (3.2) years. Among this, $80.6 \%$ were preschool children and $82.1 \%$ were urban residents. Clinically, 172 (63.9\%) and 97 (36.1\%) of them were classified as clinical stage one and two, respectively, based on WHO clinical stage criteria of HIV. From the total study participants, $89.4 \%$ were on AZT/3TC/EFV, 83.9\% did not take other drugs and $11.7 \%$ had CD4-T lymphocyte cell counts of less than or equal to 350 cells $/ \mathrm{mm}^{3}$ (Table 1).

More than one parasite was identified in 38 (13.9\%) of HAART-experienced children. Among these Ascaris lumbricoides were detected in $21(56.7 \%)$ followed by Trichuris trichiura 14 (37.8\%) and Hookworm in 2(5.4\%) of HAART-experienced children. From blood film examination, only one child was positive for Plasmodium vivax. From a review of the patient cards, 19 (6.9\%) had opportunistic infections. Among these, the highest proportion was both upper and lower respiratory tract infections and accounted for $9(47.3 \%)$ followed by pulmonary and extrapulmonary tuberculosis which accounted for 7 (36.8\%) and other generalized skin infection 3(15.9\%).

The prevalence of anemia in the current study was 31 (11.4\%). Among these16 (11.5\%) and 15 (11.2\%) were females and males, respectively. Rural resident children (20.4\%) accounted for a higher prevalence of anemia than urban $(9.4 \%)$ residents (Table 1). Based on WHO severity grading of anemia using $\mathrm{Hb}$ value, $51.6 \%$ of the study participants had moderate anemia, followed by $41.9 \%$ with 
Table I Socio-Demographic Characteristics, Associated Factors and Anemia Prevalence Among Human Immune Deficiency VirusInfected Children

\begin{tabular}{|c|c|c|c|c|c|c|}
\hline \multirow[t]{2}{*}{ Variable } & \multirow[t]{2}{*}{ Total, N (\%) } & \multicolumn{2}{|l|}{ Anemia } & \multirow[t]{2}{*}{ Chi Sq. } & \multirow[t]{2}{*}{ AOR(95\% Cl) } & \multirow[t]{2}{*}{ P-value } \\
\hline & & Yes, N (\%) & No, N (\%) & & & \\
\hline \multicolumn{7}{|l|}{ Age in years } \\
\hline$\leq 7$ & $53(19.4)$ & II (20.7) & $42(79.3)$ & 0.2 & $3(1.2-7.5)$ & 0.02 \\
\hline$>7$ & $220(80.6)$ & $20(9.1)$ & $200(90.9)$ & & I & \\
\hline \multicolumn{7}{|l|}{ Sex } \\
\hline Male & $134(49.1)$ & $15(\mid 1.2)$ & $119(88.8)$ & & & \\
\hline Female & $139(50.9)$ & $16(11.5)$ & $123(88.5)$ & 0.94 & & \\
\hline \multicolumn{7}{|l|}{ Residence } \\
\hline Urban & $49(17.9)$ & $10(20.4)$ & $39(79.6)$ & & 1 & \\
\hline Rural & $224(82.1)$ & $21(9.4)$ & $203(90.6)$ & 0.027 & $2.6(1.0-6.6)$ & 0.04 \\
\hline \multicolumn{7}{|l|}{ EDU. Status } \\
\hline NFE & $38(\mid 3.9)$ & $6(15.9)$ & $32(84.2)$ & & & \\
\hline Primary & $219(80.2)$ & $22(10.1)$ & 197(89.9) & 0.37 & & \\
\hline Secondary & $16(5.9)$ & $3(18.8)$ & $13(8 \mid .2)$ & & & \\
\hline \multicolumn{7}{|l|}{ Family size } \\
\hline$\leq 3$ & $46(16.8)$ & $8(17.4)$ & $38(82.6)$ & & & \\
\hline $4-7$ & $192(70.3)$ & $21(10.9)$ & $17 \mid(89.1)$ & & & \\
\hline$>7$ & $35(12.8)$ & $2(5.7)$ & $33(94.3)$ & 0.25 & & \\
\hline \multicolumn{7}{|l|}{ Caretaker EDU } \\
\hline No & $46(16.8)$ & $7(15.2)$ & $39(84.8)$ & & & \\
\hline Primary & $101(37)$ & $10(9.9)$ & $91(90.1)$ & 0.3 & & \\
\hline Secondary & $89(32.6)$ & $7(7.9)$ & $82(92.1)$ & & & \\
\hline College & $37(13.6)$ & $7(18.9)$ & $30(81.1)$ & 0.66 & & \\
\hline \multicolumn{7}{|l|}{ Primary caretaker } \\
\hline Mother or father & $212(77.7)$ & $24(I I .3)$ & I88(88.7) & 0.97 & & \\
\hline Other & $61(22.3)$ & $7(11.5)$ & $54(88.5)$ & & & \\
\hline \multicolumn{7}{|l|}{ Temperature $\left({ }^{\circ} \mathrm{C}\right)$} \\
\hline$\leq 37$ & $26 I(95.6)$ & 27 & 234 & 0.037 & $2.6(0.6-11.1)$ & 0.2 \\
\hline$>37$ & $12(4.4)$ & 4 & 8 & & I & \\
\hline \multicolumn{7}{|l|}{ ART Drug } \\
\hline AZT/3TC/EFV & $244(90.3)$ & $28(11.5)$ & $216(88.5)$ & & & \\
\hline ABC/3TC/CALETR & $20(3.2)$ & $\mathrm{I}(5)$ & $19(95)$ & & & \\
\hline TDF/3TC/CALETR & $9(6.5)$ & & & 0.39 & & \\
\hline \multicolumn{7}{|l|}{ Other Medication } \\
\hline No & $229(83.9)$ & $23(10.0)$ & $206(90.0)$ & & 1 & \\
\hline Cotrimoxazole & $3 I(I I .4)$ & $5(16.1)$ & $26(83.9)$ & & I.I (0.3-4.4) & 0.8 \\
\hline Other & $13(4.7)$ & $3(23.1)$ & $10(76.9)$ & 0.2 & $0.3(0.07-1.57)$ & 0.28 \\
\hline \multicolumn{7}{|l|}{ CD4 count } \\
\hline$\leq 350$ & $32(11.7)$ & $6(18.7)$ & $26(81.3)$ & $<0.001$ & & \\
\hline$>350$ & $24 I(88.3)$ & $25(10.4)$ & $216(89.6)$ & & & \\
\hline \multicolumn{7}{|l|}{ Viral load } \\
\hline$\leq 150$ & $57(20.1)$ & 14 & 43 & & I & \\
\hline$>150$ & $216(79.1 \%)$ & 17 & 199 & 0.001 & $3.4(1.36-8.3)$ & 0.009 \\
\hline
\end{tabular}

(Continued) 
Table I (Continued).

\begin{tabular}{|c|c|c|c|c|c|c|}
\hline \multirow[t]{2}{*}{ Variable } & \multirow[t]{2}{*}{ Total, N (\%) } & \multicolumn{2}{|l|}{ Anemia } & \multirow[t]{2}{*}{ Chi Sq. } & \multirow[t]{2}{*}{ AOR(95\% Cl) } & \multirow[t]{2}{*}{ P-value } \\
\hline & & Yes, N (\%) & No, N (\%) & & & \\
\hline \multicolumn{7}{|l|}{ WAZ } \\
\hline$<-2$ & $27(9.9)$ & $5(18.5)$ & $22(81.5)$ & & & \\
\hline$\geq-2$ & $246(90.1)$ & $26(10.6)$ & $220(89.4)$ & 0.18 & & \\
\hline \multicolumn{7}{|l|}{ HAZ } \\
\hline$<-2$ & $105(38.5)$ & $\mathrm{II}(10.5)$ & $94(89.5)$ & 0.85 & & \\
\hline$\geq-2$ & $168(6 \mid .5)$ & $20(11.9)$ & |48(88.I) & & & \\
\hline
\end{tabular}

Abbreviations: AOR, adjusted odds ratio; ART, antiretroviral Therapy; CD, Cluster of Differentiation; EDU, Educational; HAZ, Height to Age ratio Z score; NFR, no formal education; WHO, World Health Organization; WAZ, Weight to Age $Z$ score.

mild and $6.5 \%$ with severe anemia. The red blood cell indices and peripheral blood film examination of the participants revealed normocytic normochromic anemia was found to be more common in the current study (Figure 1).

\section{Associated Factors of Anemia}

To identify independent predictors of anemia, multivariate analysis was used and variables such as age $\leq 7(\mathrm{AOR}=3, \mathrm{CI}$ : 1.2-7.5, $\mathrm{P}=0.02)$, rural residents $(\mathrm{AOR}=2.6, \mathrm{CI}: 1.0-6.6$, $\mathrm{P}=0.042)$ and viral load $>150$ copies $/ \mathrm{mL} \quad(\mathrm{AOR}=3.4, \mathrm{CI}$; 1.36-8.3, $\mathrm{P}=0.009$ ) were strongly associated with anemia (Table 1).

\section{Discussion}

Anemia is a situation in which the hemoglobin level of an $\mathrm{RBC}$ is lesser than the reference interval for individual age, sex, location and nutritional status. ${ }^{11}$ It is the most important public health problem and a very common feature of HAARTexperienced pediatric patients. Its cause is multifactorial which may complicate the treatment and prognosis. ${ }^{17}$

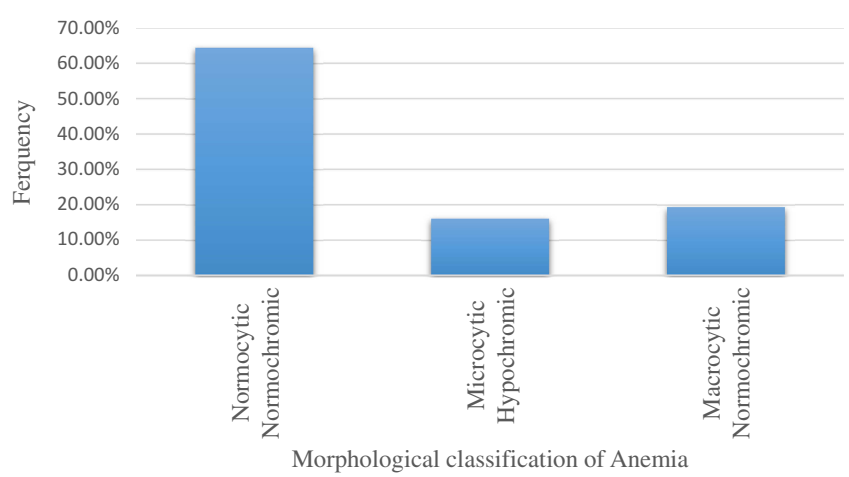

Figure I Morphological classification of anemia among human immune deficiency virus-infected children.
The total prevalence of anemia in our study was $11.4 \%$. This remains relatively comparable to the study conducted at Gondar (16.2\%). ${ }^{18}$ A similar result to our study was also reported from a comparative study done on children on HAART from Asia, Africa, and America which is $11.9 \%{ }^{19}$ On the other hand, the prevalence of anemia observed in our study was lower than the values reported in studies done at Zewditu Memorial Hospital, Addis Ababa (22.2\%), ${ }^{20}$ Jimma $(21.9 \%),{ }^{16}$ Eastern Ethiopia $(39.2 \%)^{21}$ Nigeria $(54.2 \%),{ }^{22}$ northeastern Nigeria $(24.3 \%),{ }^{23}$ western Uganda $(57.6 \%)^{9}$ and India $(47.1 \%)^{24}$. The difference in prevalence could be explained by the difference in genetic factors, geographical location (altitude), and nutritional status. The age range differences between the studies and sample size might also be another explanation for the difference between the current study and other studies because some of the studies included subjects who were between the age of 3 months and 18 years. ${ }^{9}$ Also, an additional reason for this lower prevalence could be due to the low prevalence of malaria in this study, which is the main predisposing factor of anemia among the study group. ${ }^{25,26}$ A study from Tanzania showed that malaria infection in children increases the risk of anemia. ${ }^{25}$ Different studies also support the effect of intestinal parasitosis on increasing anemia in children. ${ }^{20}$

The prevalence was higher in preschool children $(20.7 \%)$ than school children, which was comparable with the study done in eastern Ethiopia. ${ }^{21}$ Moreover, anemia was more prevalent in rural residents compared to urban dwellers. The same result was found in other parts of Ethiopia. ${ }^{20}$

The morphological examination of the current study indicated that 176 (64.5\%) participants' blood film showed normocytic normochromic anemia followed by macrocytic normochromic $53(19.4 \%)$ and microcytic hypochromic anemia $44(16.1 \%)$. Our result is in agreement with that reported in a study done in Gondar which showed that 
$46.5 \%$ of children had normocytic normochromic anemia followed by $(39.5 \%)$ of macrocytic-normochromic anemia, ${ }^{18}$ and Uganda (44.9\%) children showed microcytic-hypochromic anemia being the dominant type followed by $(26.5 \%)$ macrocytic normocytic and normocytic normochromic anemia $(19.0 \%){ }^{9}$

Based on $\mathrm{Hb}$ values, $51.6 \%$ of the study participants had moderate anemia, followed by mild (41.9\%) and severe $(6.5 \%)$. Similar findings of severe anemia were reported by studies in Nigeria $(4.8 \%),{ }^{15}$ India $(6.8 \%)^{24}$ and Uganda $(6.7 \%){ }^{9}$ On the other hand, different trends of mild, moderate and severe anemia were reported in the Northwest Ethiopia with mild (60.5\%), moderate (37.2\%) and severe $(2.3 \%)^{16}$ and Addis Ababa with mild (52.5\%) and moderate $(42.5 \%)^{20}$ and in Uganda with mild $(62.2 \%)$, moderate $(32 \%)$ and severe $(6.7 \%)$ anemia. ${ }^{9}$

There was a significant association between the age of $\leq 7$ $(\mathrm{OR}=3,95 \% \mathrm{CI}(1.2-7.5)$ at $\mathrm{P}=0.02$ which was comparable to the study reported from eastern Ethiopia $(\mathrm{AOR}=4.24,95 \% \mathrm{CI}$ (1.85-9.73) at $\mathrm{P}<0.001)^{21}$ and Uganda $(\mathrm{P}<0.001)$ which was found to be independently associated with younger age. ${ }^{9}$ This could be due to the increased development necessities of younger children, as defined by other studies ${ }^{28}$ and it could be due to different etiological causes, which comprise nutrient deficiency, immune suppression of erythropoiesis, therapeutic side effects, opportunistic infections, HIV-related malignancies, and other factors. ${ }^{11}, 29$

In this study, a viral load of $>150$ copies $/ \mathrm{mL}$ was statistically associated with anemia (AOR 3.4, 95\% CI: 1.36-8.3) which is similar to a study in Southern India which documented a viral load of $>200$ copies/mL (AOR 2.4, 95\% CI: 1.1-5.4). ${ }^{24}$ This implies that the viral load could be a risk factor for anemia. It was also associated with residency, being rural was significantly associated with anemia. This finding reflects, lack of better sociodemographic conditions such as lack of access to better feeding, health care services might contribute to the higher prevalence of anemia.

\section{Limitation of the Study}

Duration of ART drug and hepatitis B was not included as a predictor of anemia which affects the prevalence of anemia. Nutritional status and Iron diagnostic tests such as serum iron, ferritin and total iron-binding capacity (TIBC) were not considered from each child. Therefore, longitudinal studies should be conducted to estimate the cause and associated factors of anemia among HAARTexperienced children HIV-infected children.

\section{Conclusion}

The prevalence of anemia among HIV-infected HAARTexperienced children in our study was $11.4 \%$. Among these, the majority of children have moderate anemia which requires strict follow-up and monitoring to control other complications related to anemia. The factors associated with anemia in this study were age, being rural residency and viral load of $>150$ copies $/ \mathrm{mL}$. Hence, additional longitudinal studies with extended follow-up are needed to explore more on the causes of anemia and the pattern of hemoglobin changes with HAART experience in HIV-positive children in the study area.

\section{Ethics Approval and Consent to Participate}

The study was approved by the Institutional Review Board (IRB) of Jimma University College of Medicine and Health Sciences with the ethical clearance number JHRPGC/268/ 07. Consent was taken from their parents or guardians on behalf of children less than 7 years of age and ascent and written consent was received from both parents and guardians for children with the age range of 7-14 years.

\section{Abbreviations}

AIDS, Acquired Immune Deficiency Syndrome; ART, Antiretroviral Treatment; AZT, Zidovudine; CBC, Complete Blood Count; CD4+, Cluster differentiation; FACS, Fluorescence-Activated Cell Sorting; HAART, Highly Active Antiretroviral Treatment; Hgb, Hemoglobin; HIV, Human Immunodeficiency Virus; WHO, World Health Organization; HUCSH, Hawassa University Comprehensive Specialized Hospital.

\section{Data Sharing Statement}

The data that support the findings of this study are available from the corresponding author upon reasonable request with the permission of the University and the corresponding author but not publicly available.

\section{Acknowledgments}

The authors would like to thank Jimma University and Hawassa University College of Medicine and Health Science for supporting and allowing them to conduct this research. We also extend our thanks to all ART clinic staff who help us during data collection and laboratory work and $\mathrm{Mr}$. Antneh Amsalu (PhD fellow at Adelaide 
University Australia) for editing the English language. Last but not least we also thank the study participants.

\section{Author Contributions}

All authors contributed to data analysis, drafting or revising the article, gave final approval of the version to be published, and agree to be accountable for all aspects of the work.

\section{Funding}

This work was supported by Jimma University to cover transportation fees for data collectors and the cost of reagents and materials. The authors also declare that no other financial fund was secured from other sources for this research work.

\section{Disclosure}

All authors declare that they have read and approved the manuscript and ensure no competing interests.

\section{References}

1. Davies M-A, Keiser O, Eley B, et al. Outcomes of the South African national antiretroviral treatment programme for children: the IeDEA Southern Africa collaboration. South Afr Med J. 2009;99(10):730-737.

2. Quaye W, Addai-Mensah A. Prevalence of anaemia and immunological markers among Ghanaian HAART-naïve HIV-patients and those on HAART. Afr Health Sci. 2011;11(1):2-15.

3. Sliwa K. The multifactorial burden of anaemia in Africa. South Afr Med J. 2009;99(12):864-865.

4. WHO C. Worldwide Prevalence of Anaemia 1993-2005. WHO global database on anaemia; 2008.

5. Quiros-Roldan E, Castelli F, Bonito A, et al. The impact of integrase inhibitor-based regimens on markers of inflammation among HIV naïve patients. Cytokine. 2020;126:154884. doi:10.1016/j.cyto.2019.154884

6. Quiros-Roldan E, Castelli F, Lanza P, et al. The impact of antiretroviral therapy on iron homeostasis and inflammation markers in HIV-infected patients with mild anemia. J Transl Med. 2017;15 (1):256. doi:10.1186/s12967-017-1358-6

7. Gedefaw L, Yemane T, Sahlemariam Z, Yilma D. Anemia and risk factors in HAART naive and HAART experienced HIV positive persons in south west Ethiopia: a comparative study. PLoS One. 2013;8(8):e72202. doi:10.1371/journal.pone.0072202

8. Mata-Marín JA, Gaytán-Martínez JE, Martínez-Martínez RE, Arroyo-Anduiza CI, Fuentes-Allen JL, Casarrubias-Ramirez M. Risk factors and correlates for anemia in HIV treatment-naïve infected patients: a cross-sectional analytical study. BMC Res Notes. 2010;3(1):230. doi:10.1186/1756-0500-3-230

9. Ruhinda EN, Bajunirwe F, Kiwanuka J. Anaemia in HIV-infected children: severity, types and effect on response to HAART. BMC Pediatr. 2012;12(1):170. doi:10.1186/1471-2431-12-170

10. Walker AS, Doerholt K, Sharland M, Gibb DM. Response to highly active antiretroviral therapy varies with age: the UK and Ireland Collaborative HIV Paediatric Study. Aids. 2004;18(14):1915-1924. doi:10.1097/00002030-200409240-00007

11. Calis JC, van Hensbroek MB, de Haan RJ, Moons P, Brabin BJ, Bates I. HIV-associated anemia in children: a systematic review from a global perspective. Aids. 2008;22(10):1099-1112. doi:10.1097/ QAD.0b013e3282fa759f
12. Belperio PS, Rhew DC. Prevalence and outcomes of anemia in individuals with human immunodeficiency virus: a systematic review of the literature. Am J Med. 2004;116(7):27-43. doi:10.1016/j. amjmed.2003.12.010

13. Musoke PM, Mudiope P, Barlow-Mosha LN, et al. Growth, immune and viral responses in HIV infected African children receiving highly active antiretroviral therapy: a prospective cohort study. BMC Pediatr. 2010;10(1):56. doi:10.1186/14712431-10-56

14. Bunupuradah T, Kariminia A, Chan K-C, et al. Incidence and predictors of severe anemia in Asian HIV-infected children using first-line antiretroviral therapy. Int $J$ Infect Dis. 2013;17(10):e806e810. doi:10.1016/j.ijid.2013.04.006

15. Ejeliogu EU, Oguche S, Ebonyi AO, et al. Zidovudine-induced anaemia in human immunodeficiency virus infected children on highly active anti-retroviral therapy in Jos, Nigeria. $J A d v$ Med Pharm Sci. 2014. doi:10.9734/JAMPS.

16. Abebe M, Alemseged F. Hematologic abnormalities among children on Haart, in Jimma university specialized hospital, southwestern ethiopia. Ethiop J Health Sci. 2009;19(2).

17. Volberding PA, Levine AM, Dieterich D, et al. Anemia in HIV infection: clinical impact and evidence-based management strategies. Clin Infect Dis. 2004;38(10):1454-1463. doi:10.1086/383031

18. Enawgaw B, Alem M, Melku M, Addis Z, Terefe B, Yitayew G. Prevalence and associated risk factors of anemia among HIV infected children attending Gondar university hospital, Northwest Ethiopia: a cross sectional study. BMC Hematol. 2015;15(1):12. doi:10.1186/ s12878-015-0032-6

19. Firnhaber C, Smeaton L, Saukila N, et al. Comparisons of anemia, thrombocytopenia, and neutropenia at initiation of HIV antiretroviral therapy in Africa, Asia, and the Americas. Int J Infect Dis. 2010;14 (12):e1088-e1092. doi:10.1016/j.ijid.2010.08.002

20. Mihiretie H, Taye B, Tsegaye A. Magnitude of anemia and associated factors among pediatric HIV/aids patients attending Zewditu memorial hospital art clinic, Addis Ababa, Ethiopia. Anemia. 2015;2015. doi: $10.1155 / 2015 / 479329$

21. Teklemariam Z, Mitiku H, Mesfin F. Prevalence of anemia and nutritional status among HIV-positive children receiving antiretroviral therapy in Harar, eastern Ethiopa. HIV/AIDS. 2015;7: 191.

22. Ahumareze RE, Rankin J, David A, et al. Prevalence of anaemia and the relationship between haemoglobin concentration and CD4 count in HIV positive children on highly active antiretroviral therapy (HAART) in Lagos, Nigeria. Curr Pediatr Res. 2016;20:29-36.

23. Denue BA, Kida IM, Hammagabdo A, Dayar A, Sahabi MA. Prevalence of anemia and immunological markers in HIV-infected patients on highly active antiretroviral therapy in Northeastern Nigeria. Infect Dis. 2013;6:IDRT.S10477.

24. Shet A, Bhavani P, Kumarasamy N, et al. Anemia, diet and therapeutic iron among children living with HIV: a prospective cohort study. $B M C$ Pediatr. 2015;15(1):164. doi:10.1186/s12887-015-0484-7

25. Chatterjee A, Bosch RJ, Kupka R, Hunter DJ, Msamanga GI, Fawzi WW. Predictors and consequences of anaemia among antiretroviral-naive HIV-infected and HIV-uninfected children in Tanzania. Public Health Nutr. 2010;13(2):289-296. doi:10.1017/ S1368980009990802

26. Mengist HM, Taye B, Tsegaye A. Intestinal parasitosis in relation to CD4+ T cells levels and anemia among HAART initiated and HAART naive pediatric HIV patients in a model ART center in Addis Ababa, Ethiopia. PLoS One. 2015;10(2):e0117715. doi:10.1371/journal. pone. 0117715

27. Ngongondo M, Rosenberg NE, Stanley CC, et al. Anemia in people on second line antiretroviral treatment in Lilongwe, Malawi: a cross-sectional study. BMC Infect Dis. 2018;18(1):39. doi:10.1186/ s12879-018-2952-9 
28. Shet A, Mehta S, Rajagopalan N, et al. Anemia and growth failure among HIV-infected children in India: a retrospective analysis. $B M C$ Pediatr. 2009;9(1):37. doi:10.1186/1471-2431-9-37
29. Shet A, Arumugam K, Rajagopalan N, et al. The prevalence and etiology of anemia among HIV-infected children in India. Eur J Pediatr. 2012;171(3):531-540. doi:10.1007/s00431-011-1599-y

\section{Publish your work in this journal}

Drug, Healthcare and Patient Safety is an international, peer-reviewed open-access journal exploring patient safety issues in the healthcare continuum from diagnostic and screening interventions through to treatment, drug therapy and surgery. The journal is characterized by the rapid reporting of reviews, original research, clinical, epidemiological and post-marketing surveillance studies, risk management, health

Submit your manuscript here: https://www.dovepress.com/drug-healthcare-and-patient-safety-journal literacy and educational programs across all areas of healthcare delivery. The manuscript management system is completely online and includes a very quick and fair peer-review system. Visit $\mathrm{http}: / / \mathrm{www}$.dovepress.com/testimonials.php to read real quotes from published authors. 